\title{
Theranostics in Brain Tumors
}

\author{
Hossein Shooli, MDa, Reza Nemati, MD ${ }^{b}$, Hojjat Ahmadzadehfar, MD, MSc ${ }^{c}$, \\ Mariam Aboian, MD, PhD ${ }^{d}$, Esmail Jafari, MSc ${ }^{a}$, Narges Jokar, MSc ${ }^{a}$, Iraj Nabipour, MD ${ }^{e}$, \\ Habibollah Dadgar, MSc ${ }^{f}$, Ali Gholamrezanezhad, MD, FEBNM, DABR ${ }^{g}$, Mykol Larvie, MD, PhD ${ }^{\text {h }}$, \\ Majid Assadi, MD, FASNC ${ }^{a, *}$
}

\section{KEYWORDS}

$\bullet$ Theranostics • Brain tumor $\bullet$ PET/CT • PET/MR imaging $\bullet$ Neuro-oncology $\bullet$ Neurotheranostics

- Radioneurotheranostics

\section{KEY POINTS}

- Theranostic nuclear oncology, mainly in neuro-oncology (neurotheranostics), aims to combine cancer imaging and therapy using the same molecular targeting platform.

- The ability of radioneurotheranostic agents to interact with cancer cells at the molecular level with high specificity can significantly improve the effectiveness of cancer therapy and helps to identify patients who are most likely to benefit from tumor molecular radionuclide therapy.

- A variety of biologic targets are under investigation for treating brain tumors.

- PET-based precision imaging can substantially improve the therapeutic efficacy of radiotheranostic approach in brain tumors.

\section{INTRODUCTION}

Brain tumors are divided into primary brain tumors, which originate from the brain itself, and metastatic brain tumors, which originate from the tumors of other body parts. According to the World Health Organization (WHO) grading system, primary brain tumors are classified as low-grade tumors (grade I-II) and high-grade tumors (grade III-IV). Furthermore, the most common metastatic brain tumors originate from the breast, lung, and skin (melanoma). ${ }^{1}$ Among the high grade gliomas, there is differentiation based on origin of the tumor, such as secondary high grade gliomas are transformed from lower grade gliomas. On the other hand, primary glioblastomas commonly do not have a precursor lesion. ${ }^{2}$ Among primary brain gliomas, glioblastoma (GBM) is the most frequent and most lethal primary brain tumor.

Conflicts of interest: The authors declare that they have no commercial or financial conflict of interest. Financial disclosures: National Center for Advancing Translational Science (NCATS), CTSA grant KL2 TR001862 to Mariam Aboian. Other authors have nothing to disclose.

a Department of Molecular Imaging and Radionuclide Therapy (MIRT), The Persian Gulf Nuclear Medicine Research Center, Bushehr Medical University Hospital, School of Medicine, Bushehr University of Medical Sciences, Moallem St, Bushehr, Iran; ${ }^{b}$ Department of Neurology, Bushehr Medical University Hospital, Bushehr University of Medical Sciences, School of Medicine, Bushehr, Iran; ${ }^{c}$ Department of Nuclear Medicine, Klinikum Westfalen, Dortmund, Germany; ${ }^{d}$ Department of Radiology, Yale University School of Medicine, New Haven,

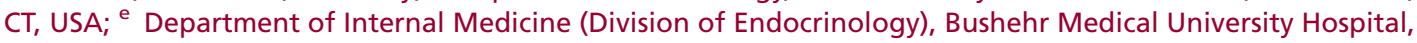
The Persian Gulf Tropical Medicine Research Center, The Persian Gulf Biomedical Sciences Research Institute, Bushehr University of Medical Sciences, Bushehr, Iran; ${ }^{\dagger}$ Cancer Research Center, RAZAVI Hospital, Imam Reza International University, Mashhad, Iran; ${ }^{9}$ Department of Diagnostic Radiology, Keck School of Medicine, University of Southern California (USC), 1520 San Pablo Street, Suite L1600, Los Angeles, CA 90033, USA;

${ }^{\mathrm{h}}$ Department of Radiology, Cleveland Clinic, Cleveland, OH, USA

* Corresponding author. Department of Molecular Imaging and Radionuclide Therapy, The Persian Gulf Nuclear Medicine Research Center, Bushehr Medical University Hospital, School of Medicine, Bushehr University of Medical Sciences, Moallem St, Bushehr, Iran 7514633341.

E-mail addresses: assadipoya@yahoo.com; asadi@bpums.ac.ir 